\section{Regression, lineare}

R.-D. Hilgers ${ }^{1}$, N. Heussen ${ }^{1}$ und S. Stanzel ${ }^{2}$

${ }^{1}$ Institut für Medizinische Statistik, Universitätsklinikum der RWTH Aachen, Aachen, Deutschland

${ }^{2}$ DKFZ Heidelberg, Heidelberg, Deutschland

\section{Englischer Begriff linear regression}

Definition Lineare Regression beschreibt einen linearen funktionalen Zusammenhang zwischen einer abhängigen Größe und einer oder mehreren unabhängigen Größen.
Ein wichtiger Spezialfall der linearen Regression ist die einfache lineare Regression mit der $>$ Regressionsfunktion $\mathrm{Y}=\mathrm{a}+\mathrm{bX}$. Bei der Darstellung dieser Funktion in einem Koordinatensystem ergibt sich eine Gerade, die sog. $>$ Regressionsgerade.

\section{Literatur}

Rasch D (1988) Biometrisches Wörterbuch. Verlag Harri Deutsch, Frankfurt am Main 